\title{
Microstructural analysis of crack growth caused by static and dynamic loads in a carbon fiber reinforced cement paste
}

\author{
Matthias Rutzen \& Dirk Volkmer* \\ Chair of Solid State Chemistry, University of Augsburg, Augsburg, Germany \\ *Corresponding author email: dirk.volkmer@physik.uni-augsburg.de \\ Ursula Weiß, Lisa Reischmann \& Malte A. Peter \\ Research Unit Applied Analysis, University of Augsburg, Augsburg, Germany \\ Philipp Lauff \& Oliver Fischer \\ Chair of Concrete \& Masonry Structures, Technical University Munich, Munich, Germany \\ Manuel Raith \& Christian Große \\ Chair of Non-Destructive Testing, Technical University of Munich, Munich, Germany
}

ABSTRACT: By using a novel fiber-reinforced cement paste in a 3D-printing process, a material with vastly improved flexural and tensile strength can be created. The extrusion-based process leads to a high degree of fiber orientation. A fiber content of 3 vol. \% results in static flexural strengths of above $100 \mathrm{MPa}$. High-strength fiber-reinforced materials have potential to be used in lightweight construction. Due to the susceptibility of lightweight structural members to oscillating dynamic loads, cyclic flexural and tensile tests with up to 10 million cycles are carried out. To characterize the complex (micro-)cracking behavior of the material, the tests are augmented by external deformation sensors (strain gauges, fiber-optic sensors, photogrammetry) and microstructural analysis (X-ray CT, acoustic emission sensors, coda-wave interferometry). The experimental data will serve as the basis for multiscale-modelling approach which uses representative volume elements created from high-resolution CT scans.

\section{INTRODUCTION}

Cementitious materials show strong performance when loaded under compression, but are relatively weak when tensile or flexural loading regimens are applied. This weak point can be greatly reduced when materials that show high tensile strength are introduced to the cement matrix. Traditionally, this is done with reinforcing steel; recently however, fiber reinforcement has been of great scientific interest due to its potential in the field of lightweight structures. A novel concept using a carbon fiber reinforced mortar in a Liquid Deposition Modelling (LDM) process leads to a cementitious material with a nearly unidirectional fiber alignment (Hambach \& Volkmer 2017). LDM is a 3D-printing method in which a liquid material is extruded layer by layer until its final shape is achieved. The material hardens either during or after the process. If a fiber-reinforced mortar is extruded through a small nozzle, the fibers will align themselves along the nozzle's direction of movement. A content of 3 volume percent of oriented fibers results in a static flexural strength of above $100 \mathrm{MPa}$ (Hambach et al. 2016). Using this technique, a specific stress profile for a structural member can be matched to the nozzle path to optimize fiber alignment to the expected load. Figure 1 shows typical stress-strain diagrams of small 3-point bending beams reinforced with various amounts of carbon fibers, aligned in the direction of the tensile stress occurring during testing.

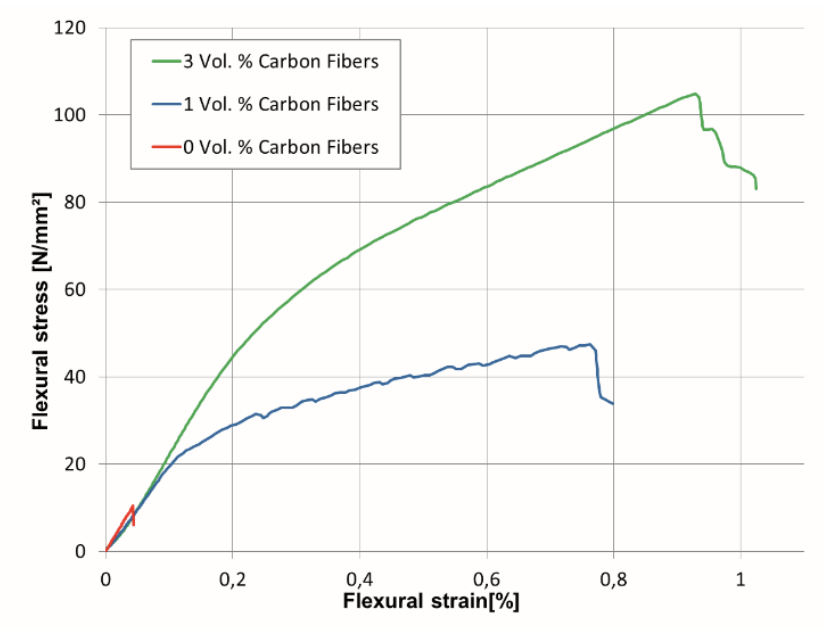

Figure 1. Stress-strain behavior during 3-point bending for reinforced mortar with various amounts of chopped carbon fibers admixed. The fibers are oriented in the direction of the tensile stress.

As can be seen, even relatively small amounts of correctly aligned fibers lead to an extraordinary increase in ultimate flexural strength and toughness. 
Mechanical behavior can be subdivided into a linearelastic phase at lower stress levels and a strain-hardening phase at higher stress levels. The strain-hardening phase is characterized by the formation of multiple micro-cracks in the cementitious matrix which are bridged by the fibers until total fracture (Kanada \& Li 1998; Jun \& Mechtcherine 2010). A side-view of a sample after final failure can be seen in Figure 2. Around a fatal crack with relatively large crack width, many smaller hairline cracks are visible. The image was taken on a Zeiss Smartzoom 5 digital microscope; the cracks were colored red using GIMP 2.8.20.

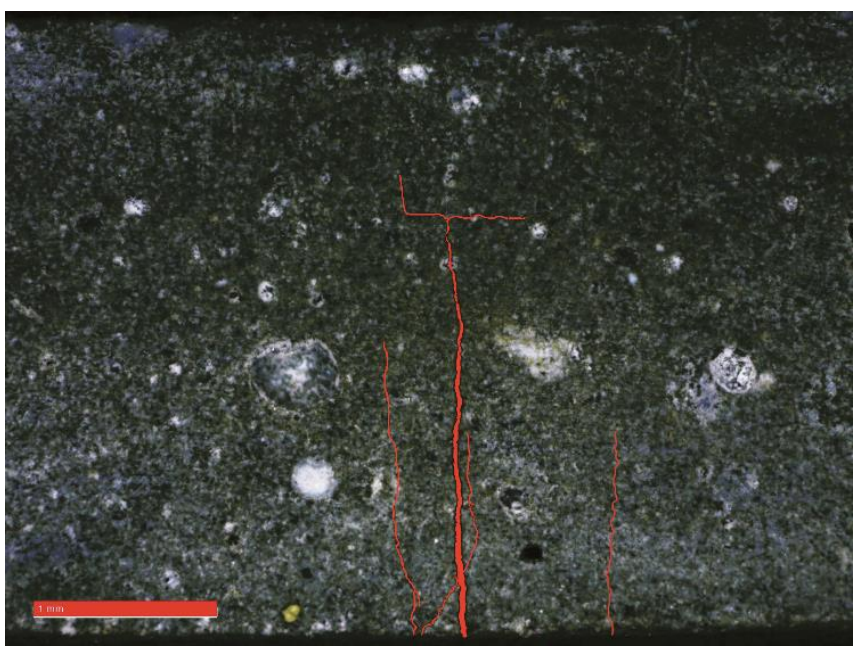

Figure 2. Side-view on a miniature beam specimen after failure in a 3-point bending test. Micro-cracks were colored red using digital post-processing.

These micro cracks are not adequately described as integral deformation on the macroscopic scale. Instead, a multitude of measuring techniques suitable for capturing localized straining phenomena or finely detailed damage indicators (X-ray CT, digital microscopy, fiber-optic sensors, photogrammetry, acoustic emission analysis, etc.) are used to more accurately describe the sample's behavior.

While this is also applicable to static tests, the following research wants to explore the influence of aligned carbon fibers on resistance against cyclic loading, in particular. As structural members with fiber reinforcement have great potential for use as lightweight elements with minimal wall thickness, susceptibility to damage by oscillating forces increases - thus, gaining understanding of the particular mechanisms of these processes is critical.

The data gathered during the experimental phase are also used for a multiscale modelling approach, which combines a description of a macroscopic workpiece with that of fine structures on the mesoscale with the help of representative volume elements (RVE).

\section{MICROSTRUCTURAL ANALYSIS OF MINIATURE BEAM SPECIMENS UNDER FLEXURAL LOAD}

\subsection{Sample preparation}

The experiments were carried out using a fiber-reinforced cement paste recipe described by Hambach $\&$ Volkmer (2016). A dry mix of 61,5 wt\% CEM I 52,5 R (Schwenk Zement KG) and $21 \mathrm{wt} \%$ silica fume EFACO (Egyptian Ferro Alloys Company SAE) was mixed with $15 \mathrm{wt} \%$ of deionized water and 2.5 wt $\%$ water-reducing agent Glenium ACE 430 (BASF SE) using a Heidolph Hei-Torque 400 overhead mixer at $400 \mathrm{rpm}$. After homogenization of all ingredients, the carbon fibers are added (Toho Tenax J HT C261, length: $3 \mathrm{~mm}$, diameter $7 \mu \mathrm{m}$, tensile strength: $3950 \mathrm{MPa}$, Young's modulus: $230 \mathrm{GPa}$ ) and mixed at $60 \mathrm{rpm}$ until no fiber agglomerates are visible anymore.

The cement is then filled into a $20 \mathrm{~mL}$ disposable syringe (B. Braun-Melsungen AG) and extruded into a teflon mold of $60 \mathrm{~mm} \times 13 \mathrm{~mm} \times 3 \mathrm{~mm}$. The samples were stored in a desiccator over deionized water (100\% relative humidity) for $24 \mathrm{~h}$ for hardening. After this period, the samples were put in a bath of deionized water for 6 days and finally stored for another 21 days in a desiccator over an oversaturated sodium bromide solution (60\% relative humidity). After 7 days storage, the specimens were cut into strips of $60 \mathrm{~mm} \times 3 \mathrm{~mm} \times 3 \mathrm{~mm}$ using a Buehler IsoMet low-speed saw.

\subsection{Examination of fracture zones using $X$-ray $C T$}

As a first step, the material's behavior under static flexural load is investigated. The strength values thus obtained will also serve as a reference for the stress levels applied during the following cyclic loading experiments. Testing is done on beam-shaped samples with dimensions of $60 \mathrm{~mm} \times 3 \mathrm{~mm} \times 3 \mathrm{~mm}$ in a 3point bending setup until failure using a Zwick/Roell Zwicki-Line Z0.5 Universal Testing Machine with a $5 \mathrm{kN}$ load cell attached. After failure, the area next to the fatal crack is scanned using X-ray CT.

The small cross-section of $3 \mathrm{~mm} \times 3 \mathrm{~mm}$ allows for a high-resolution CT scan with no further preparation; thus, no further damage is introduced to the sample. Using a GE nanotom $\mathrm{m}$ CT-scanner, voxel sizes between $1.5 \mu \mathrm{m}$ and $2 \mu \mathrm{m}$ were typically achieved. One scan consists of 2000 single images taken with a voltage of $190 \mathrm{kV}$ and a current of 70 $\mu \mathrm{A}$. Reconstructions were carried out using the software phoenix dataos $\mid \mathrm{x}$, ring-shaped artefacts were removed automatically during post-processing. The reconstructed images were viewed and processed in VGStudio Max 2.1. Figure 3 shows a CT-scan of a beam sample after static failure in a 3-point bending 
test. Grayscale values corresponding to fibers are marked in red.

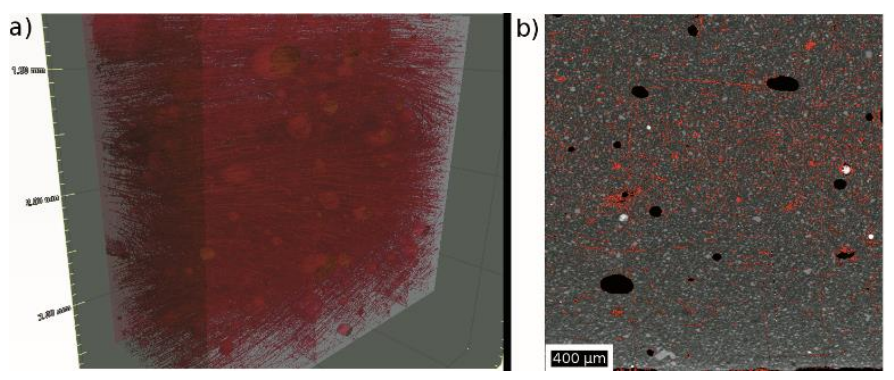

Figure 3. CT-scan of a sample reinforced with carbon fiber Tenax J HT C261(diameter of $7 \mu \mathrm{m}$ ) after a static 3-point bending test. Grayscale values corresponding to fibers and voids are marked in red. a) 3D isosurface of fibers and voids within the sample. b) Region of interest from a tomographic slice. Fibers can be seen as thin lines oriented in the horizontal direction, hairline cracks are visible as very thin lines in the vertical direction.

As can be seen in Figure 3b, fibers, cracks, and pores are visible to the human eye; however, due to overlapping greyscale values, the segmentation also produces lots of single particles which can be considered noise. Thus, selecting a range of grayscale values to separate the different materials is alone not a viable segmentation strategy for this material. Current investigations are underway to find suitable image filters to reduce noise in the original image (e.g. by means of binarization), as well as post-segmentation algorithms to divide segmented structures by geometric parameters like particle size or aspect ratio. A wellsegmented image will allow for powerful quantitative analysis of microstructural parameters such as pore volume, fiber alignment histograms, and crack length.

Once the segmentation procedure reaches a satisfactory standard, samples will be investigated for damage caused by cyclic load in an ex-situ method. A sample is put under cyclic load using a TA Instruments Q800 Dynamic-Mechanical Analyzer. After a certain number of load cycles it is scanned using XRay CT. Once the scan is complete, the sample undergoes a cyclic loading regime again and the process is repeated until the sample fails. This way, observing the growth of specific micro-cracks in the loaded area as well the development in other microstructural changes should be possible. This information then allows conclusions to be drawn regarding the mechanisms behind crack propagation in fiber-reinforced cementitious composites.

\section{$2.3 X$-ray CT as a basis for creation of representative volume elements}

In addition to microstructural analysis, the $\mathrm{CT}$ data are meant to allow for the creation of representative volume elements (RVE) for use in multiscale numerical modeling. Since carbon fibers are very small in scale compared to a macroscopic sample, a simulated workpiece needs to use these RVE to properly reflect the influence of mesoscale particles. To allow for easier segmentation, carbon fibers with a wider diameter (Kreca Chopped Fiber Type C-103 T, Kureha GmbH, diameter: $16.3 \mu \mathrm{m}$, length: $3.1 \mathrm{~mm}$, tensile strength: $875 \mathrm{MPa}$, Young's modulus: $35 \mathrm{GPa}$ ) were used. The reconstructed scan can be seen in Figure 4.
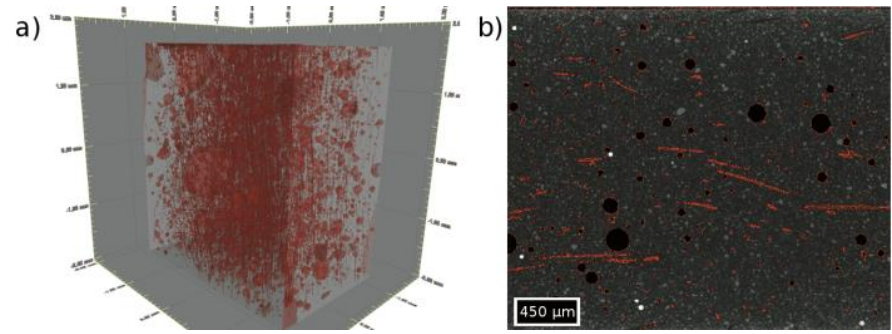

Figure 4. CT-scan of a sample containing carbon fiber Kreca C$103 \mathrm{~T}$ (diameter of $16.3 \mu \mathrm{m}$ ), serving as a basis for RVEs. Grayscale values corresponding to fibers and voids are marked in red. a) 3D isosurface of fibers and voids within the sample. b) Tomographic slice showing segmented fibers and voids marked in red.

As Figure 4 shows, there is much less noise included in the final segmentation due to the coarser nature of the fibers. While those fibers are inferior in mechanical properties, and thus not suitable as reinforcement material, they provide a valuable starting point in the creation of an RVE for modelling purposes, since factors like fiber alignment angles have not visibly changed while the quality of the segmentation is much better.

\section{HIGH-RESOLUTION SURFACE DEFORMATION MEASUREMENTS ON MACROSCALE BONE-SHAPED SPECIMENS UNDER UNIAXIAL TENSION}

Testing of uniaxial tensile loads is done on macroscale bone-shaped specimens. For sample preparation, a 3D printer was developed enabling precise positioning of the extruded strands, thus ensuring proper fiber orientation. For pumping concrete, different pumping methods were investigated, as special demands were placed on the pumping system due to high viscosity and strong cross-sectional taper of the nozzle. Another necessary feature is a fast response time to track speed changes so that not too much or too little material is extruded during acceleration and deceleration ramps. The best results could be achieved using a pneumatic piston pump which compresses a foil cartridge. The printing path during production of the specimens is adjusted in such a way as to densify the single strands as tightly together as possible, avoiding macroporosity. 


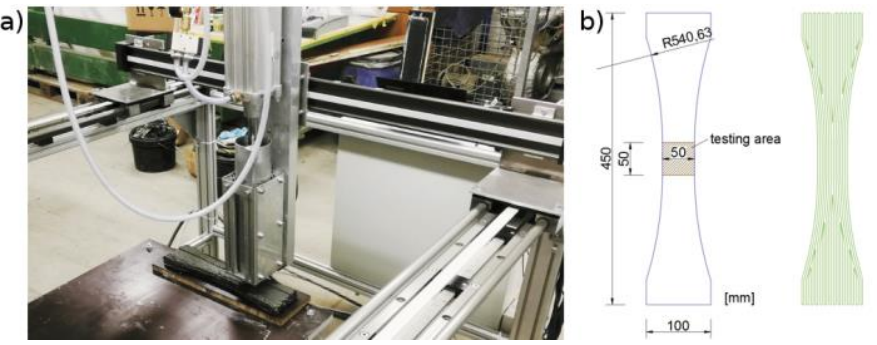

Figure 5. 3D printer for the production of cementitious materials printing tensile specimen (a), dimensions of the specimen and the nozzle path used (b)

To this end, many nozzle paths are theoretically viable. As shown in Figure 5, the circular outlines of the specimen require a path that assures smooth and equal curvature on both sides of the specimen. Jagged edges in the load-bearing zone may lead to stress peaks and early specimen failure during fatigue testing. Therefore, different nozzle paths were constructed and examined to minimize matrix heterogeneity and macroporosity in between printed strands.

Next to the specimen shown in Figure 5, there will be tests on smaller ones which are also bone-shaped but only $160 \mathrm{~mm}$ in length to investigate the influence of size. During the whole research project, different carbon fiber contents, swinging speeds, and load levels from 20 to $80 \%$ with a bottom to top load ratio of $20 \%$ of short term resistance will be applied. One of the project's main aspects is to examine the behavior in construction-practical stress levels and to determine the residual strength after previous high-cyclic loading. For this purpose, Wöhler tests will be aborted early during phase II (stable crack growth) and III (unstable crack growth) on the basis of observed damage levels on the sample and the remaining load-bearing capacity will be determined. With this information, we hope to be able to predict the transition from stable to unstable crack growth and on this basis gain access to improved life-cycle analysis of the material.

If static and dynamic tensile tests are carried out with the ends of the specimen tightly clamped, cracking of the samples will lead to eccentricities in the testing setup. Using a loose-jointed connection however, will allow for a repositioning of the specimen in a way that the cracked sample will still be loaded centrically. Based on the primary and non-cracked profile, there is a bending load which cannot be determined by our strain measurement setup. Therefore, the specimen is glued with duromer adhesive MCBauchemie MC-DUR 1280 to the bearing plates connected to the servo-hydraulic testing machine EVO 1000.

This research aims to closely observe the crack process while working with non-notched specimens. This can be achieved with high-resolution surface measurement techniques like fiber optical sensors and photogrammetry or non-destructive testing methods like acoustic emission analysis and ultrasonic based coda wave interferometry.

In the fiber optic measurement, glass fibers are glued onto the specimen surface and scanned at short intervals using a laser beam. Defects, density, and geometry fluctuations in the glass fiber cause reflection of the laser beam (Rayleigh backscatter). Deformation of the glass fiber changes the wavelength of the light. This, by proxy, makes it possible to measure the actual strain of the sample. The distance between individual measuring points is only a few millimeters, so that a very detailed statement of the strain profile in the direction of the glass fiber can be determined, as shown in Figure 6. (Schmidt-Thrö, Scheufler \& Fischer (2016))

Photogrammetry is based on the observation of surface deformation by means of a stereo camera. Especially at low camera-object distances, very precise pixel shifts can be recorded, allowing us to calculate the strain on the specimen's surface (see Figure 6). In addition, this measurement method operates without contact, which means that no faulty measurements through the connection (adhesive) can occur.
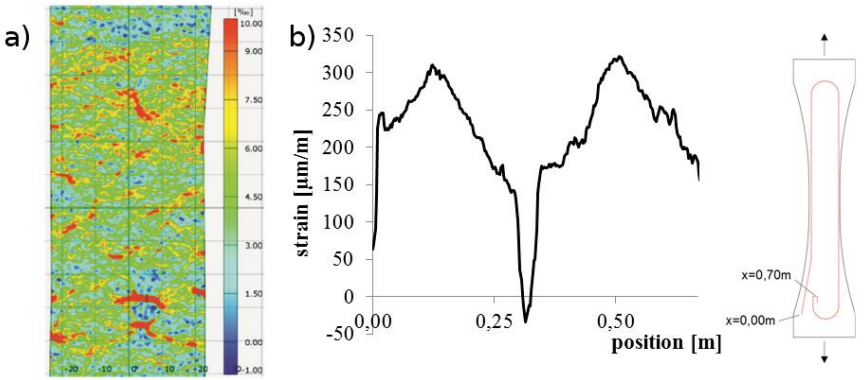

Figure 6. Micro-crack analysis with surface strain measurement, i.e. photogrammetry (a) and fiber optical sensor (b)

In addition to the methods of near-surface strain measurement, methods that provide information about the entire sample volume are very useful. A proven and well documented method for this purpose is sound emission analysis. After a certain point, deforming a brittle material will result in the failure of single weak zones inside its volume. This can be measured as a release of energy in the form of mechanical waves. These mechanical waves can be registered on the surface and thus provide an insight into the underlying damage mechanism. Before the acoustic emission experiments, the compressional wave velocity must be determined. In order to do this, a scanning ultrasound measurement method was applied on two material samples. A dense spatial sampling of 1 $\mathrm{mm}$ yields a compressional wave velocity of $\mathrm{c}_{\mathrm{p}}=$ $4605 \pm 40 \mathrm{~m} / \mathrm{s}$ and a surface wave velocity $\mathrm{c}_{\mathrm{r}}=2357$ $\pm 3 \mathrm{~m} / \mathrm{s}$. The resulting wave-field is shown in Figure 7. Based on these wave velocities, the dynamic elastic modulus was computed to $\mathrm{E}=29891 \pm 450 \mathrm{~N} / \mathrm{mm}^{2}$. 


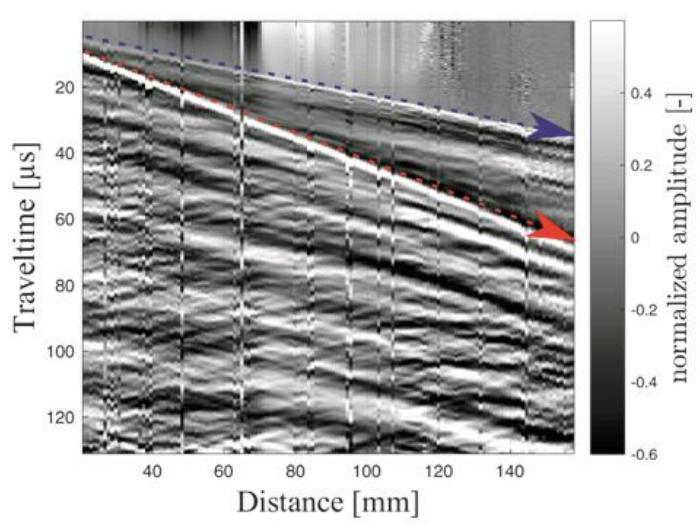

Figure 7. Result from a B-Scan measurement. The incident compression wave is shown by the blue arrow and the surface wave is highlighted by the red arrow.

The modulus of elasticity and wave propagation velocities are sensitive to development of microcracks (Thiele 2018). Therefore, future cyclic loading experiments will be monitored using ultrasound transmission measurements in order to capture the development of micro cracks and elastic parameters over time.

\section{MULTISCALE NUMERICAL SIMULATION}

The numerical simulation of the mechanical behavior of the fiber-reinforced cement paste requires a multiscale approach taking into account the scale of the carbon fibers besides the specimen scale. First tests have shown that aggregates and pores do not need to be resolved explicitly for this material. Therefore, in the simulation, we consider the macroscopic workpiece and resolve the mesoscale (i.e. the carbon fiber) by representative volume elements (RVE). The computational-homogenization approach (see Zohdi \& Wriggers 2005, Charalambabis 2010 or Geers et al. 2010) then requires a localized determination of the material law in each macroscopic point with the help of the solution of cell problems posed in the RVE at that point. Specifically, the macroscopic relation between stress tensor $\sigma$ and deformation gradient $\nabla \mathrm{u}$ is given by averages of properties calculated in the RVE leading to a numerical material law of the form

$$
\sigma=\mathrm{f}(\nabla \mathrm{u})
$$

where the mapping f resolves the macro-micromacro transition and represents the local effective material law.

To achieve a realistic model, the RVEs are taken from actual X-ray CT data (cf. Section 2.3). For this purpose, the carbon fibers are identified in a typical reconstructed CT-data scan and converted into fiber centerlines. To transfer these centerlines into a proper finite-element mesh, they are exported to a finite-element software and are expanded cylindrically to achieve a voluminous body with a diameter matching that of the fiber to be simulated. The extreme aspect ratio of the carbon fibers and the fact that they are mostly of unidirectional alignment requires the use of long cuboid RVEs.

Figure 8 shows a representative volume element of size $950 \mu \mathrm{m} \times 950 \mu \mathrm{m} \times 3670 \mu \mathrm{m}$ extracted from $\mu$ CT-data (Figure 8a) and reconstructed in Comsol Multiphysics (Figure 8b) for fiber-reinforced cement paste with carbon fibers of $16.3 \mu \mathrm{m}$ diameter and 3.1 $\mathrm{mm}$ length. The number of carbon fibers (whole or parts thereof) contained in the RVE is 130. The full specimen from which the RVE was extracted is 3.33 $\mathrm{mm} \times 2.44 \mathrm{~mm} \times 3.97 \mathrm{~mm}$ in size and the volume fraction of carbon fibers is $1.08 \%$. The resolution of the $\mu$-CT-data is given by a voxel side length of 1.67 $\mu \mathrm{m}$.
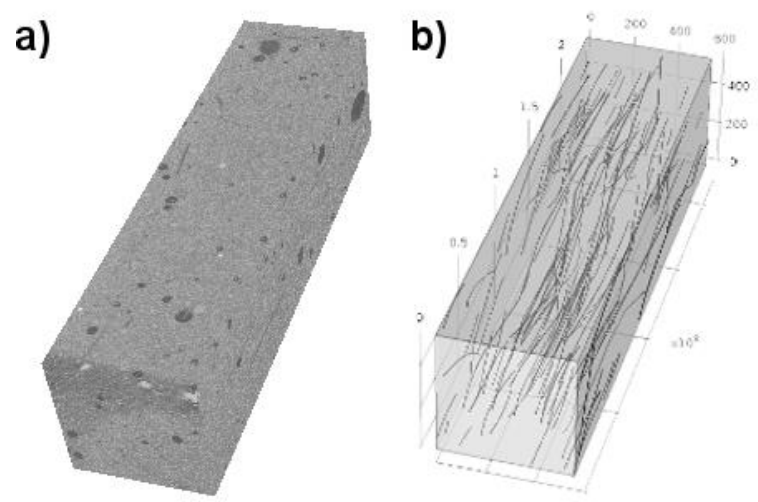

Figure 8. RVE of size $950 \mu \mathrm{m}$ x $950 \mu \mathrm{m}$ x $3670 \mu \mathrm{m}$ : a) X-rayCT-scan, b) reconstruction in Comsol Multiphysics. The single fibers of the RVE, oriented in z-direction, were extracted from $\mu$-CT-data and imported in Comsol Multiphysics via Matlab LiveLink.

Figure 9 shows a cross-section of the RVE depicted in figure 8 normal to the $y$-axis of $\mu$-CT-data (Figure 9a) and reconstructed data (Figure 9b). Figure 9a shows a cross-section of the RVE as an image with depth of one voxel. Figure $9 b$ illustrates the same cross-section of the reconstructed data. In order to achieve visual comparability, a corresponding slice with depth $33.4 \mu \mathrm{m}$ (about 20 voxels) is cut out of the reconstructed RVE in Comsol.

a)

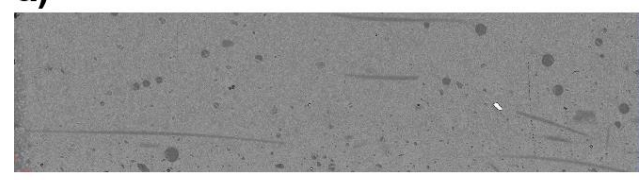

b)

Figure 9. Cross-section of the RVE a) from $\mu$-CT-data and b) reconstructed in Comsol Multiphysics. 
Numerical simulations based on this approach and comparisons with experimental data are currently underway. As a future step, it is planned to incorporate hairline cracks as observed in specimens having undergone cyclic loading and comparing the simulated mechanical properties with the experimentally measured data in order to gain deeper insight into the influence of microscopic cracks on the durability of the carbon fiber reinforced cement paste.

\section{CONCLUSIONS}

The use of short carbon fiber reinforced composites in 3D printing holds great potential for innovative lightweight construction methods. However, additional attention has to be paid to fatigue behavior. The highly effective use of carbon fibers produces a high-performance material that exceeds other cementitious materials in terms of tensile strength by a multitude.

In order to investigate the fatigue behavior and especially the crack development of this material, complex examinations on localized strain and microstructural changes were carried out. Especially high resolution X-ray $\mathrm{CT}$, surface strain measurements based on image correlation and fiber optics, as well as acoustic emission analysis are mentioned. The main focus lies on understanding local crack development and material degradation. The knowledge gained is then translated into mathematical models to predict the point of failure. Finally, the numerical results are reconciled with measured data, so that the micro-macro-micro relationship cycle is closed.

\section{ACKNOWLEDGEMENTS}

The authors thank Schwenk Zement KG for their kind supply of Portland Cement. This research work is made possible by and as part of SPP 2020: Cyclic deterioration of High-Performance Concrete in an experimental-virtual lab, funded by DFG. The authors would like to give their thanks to everyone involved.

\section{REFERENCES}

Charalambakis, N. (2010): Homogenization Techniques and Micromechanics. Survey and Perspectives; Appl. Mech. Rev. 63

Geers, M.G.D.; Kouznetsova, V.G.; Brekelmans W.A.M. (2010): Multi-scale computational homogenization: trends and challenges; J. Comp. Appl. Math 234, pp. 2175-2182

Hambach, M.; Möller, H.; Neumann, T.; Volkmer, D. (2016): Portland cement paste with aligned carbon fibers exhibiting exceptionally high flexural strength $(>100 \mathrm{MPa}$ ); Cement and Concrete Research 89, pp. 80-86
Hambach, M.; Volkmer, D. (2017): Properties of 3D-printed fiber-reinforced Portland cement paste; Cement and Concrete Composites 79, pp. 62-70

Jun, P.; Mechtcherine V. (2010): Behaviour of Strain-hardening Cement-based Composites (SHCC) under monotonic and cyclic tensile loading, Part 1 - Experimental Investigations; Cement \& Concrete Composites 32, pp. 801-809

Kanada T., Li V.C.(1998); Multiple Cracking Sequence and Saturation in Fibre Reinforced Cementitious Composites; Concrete Research and Technology 9

Schmidt-Thrö, G.; Scheufler, W.; Fischer, O. (2016): Kontinuierliche faseroptische Dehnungsmessung im Stahlbetonbau. Beton- und Stahlbetonbau, Vol. 111, pp. 496-504

Zohdi, T. I.; Wriggers, P. (2005): An Introduction to Computational Micromechanics, Lecture Notes in Applied and Computational Mechanics, Volume 20, Corrected Second Printing, Springer, ISBN: 978-3-540-77482-2 